\title{
Adhesive Bonding to Dentin Improved by Polymerizable Cyclodextrin Derivatives
}

Rafael L. Bowen, Gary E. Schumacher, Anthony A. Giuseppetti

Paffenbarger Research Center, American Dental Association Foundation, Gaithersburg, MD 20899-8546

\section{Charles M. Guttman}

Polymers Division National Institute of Standards and Technology, Gaithersburg, MD 20899-0001

\section{Clifton M. Carey}

Paffenberger Research Center, American Dental Association Foundation, Gaithersburg, MD 20899-8546

ray.bowen@nist.gov schumacher@nist.gov giuseppetti@nist.gov charles.guttman@nist.gov clifton.carey@nist.gov

\begin{abstract}
The objective of this work was to determine bonding characteristics of a hydrophilic monomer formulation containing polymerizable cyclodextrin derivatives. The hypothesis was that a formulation containing hydrophilic cross-linking diluent comonomers and cyclodextrins with functional groups attached by hydrolytically stable ether linkages could form strong adhesive bonds to dentin. The previously synthesized polymerizable cyclodextrin derivatives were formulated with sorbitol dimethacrylate, methacrylic acid and phenylbis(2,4,6-trimethylbenzoyl) phosphine oxide photoinitiator. The same formulation without the polymerizable cyclodextrin derivatives isolated the effects of the polymerizable cyclodextrin derivatives. A commercial self-etching bonding system was tested as a comparative control. Ground mid-coronal dentin was etched with $37 \%$ phosphoric acid $\left(\mathrm{H}_{3} \mathrm{PO}_{4}\right)$ for $15 \mathrm{~s}$ and rinsed with distilled water for $10 \mathrm{~s}$. Formulations were applied to the moist dentin and light-cured $10 \mathrm{~s}$. A packable composite was then applied through irises and light-cured $60 \mathrm{~s}$. Teeth were stored in water for $24 \mathrm{~h}$ before bonds were tested in a shearing orientation. One-way ANOVA was performed on the data. The average values of shear bond strengths were defined as loads at fracture divided by the $4 \mathrm{~mm}$ diameter iris areas. The average value of shear bond strength for the
\end{abstract}

formulation containing the polymerizable cyclodextrin derivatives was higher $(\mathrm{p}<0.05)$, where $\mathrm{p}$ is a fraction of the probability distribution) than that of the same monomeric formulation except that the polymerizable cyclodextrin derivatives were not included. This was supporting evidence that the polymerizable cyclodextrin derivatives contributed to improved bonding. The average value of shear bond strength for the formulation containing the polymerizable cyclodextrin derivatives was also higher $(p<0.05)$ than that of the commercial self-etching bonding system. These preliminary results are in accordance with the hypothesis that formulations containing polymerizable cyclodextrin derivatives can form strong adhesive bonds to hydrated dentin surfaces. Further improvements in bonding to hydrated biological tissues by use of advanced formulations are anticipated.

Key words: bond strengths; cross-linking comonomers for polymerizable cyclodextrin derivatives; dental adhesive bonding formulations.

Accepted: January 5, 2009

Available online: http://www.nist.gov/jres

\section{Introduction}

Because most dentists spend much of their time restoring teeth that have developed what appear to be recurrent caries at the margins of previous restorations [1], improved bonding materials and application methods are needed to restore teeth esthetically in a manner that will prevent formation of staining and other interfacial defects. It appears that none of the currently available adhesive compositions and their instructions for use is ideal for bonding caries-preventive resins or contemporary composite restorative 
materials to dental enamel and, especially, to dentin [2]. Replacements of sealants and composite restorations are most often a result of interfacial separations between tooth surfaces and the applied resins, with resulting discolorations and the effects of harbored microorganisms. The present report describes the testing of novel materials with the objective of providing improvements in bonding capabilities.

One of the formulations evaluated here includes a family of monomers referred to as polymerizable cyclodextrin derivatives $[3,4]$. Most of the diverse members of this family each contain polymerizable groups, carboxylate-terminated ligand (surface-binding) groups, and residual hydroxyl groups (Fig. 1.). The hydrophobic copolymerizable groups are posited to copolymerize with diluent comonomers and other resin-based materials. The polymerizable cyclodextrin derivatives' hydrophilic carboxylate ligand groups can form ionic, surface-binding interactions with the embedded dental collagen and also with the calcium phosphate phases of dentin and enamel. Their residual hydroxyl groups will form electrostatic hydrogen bonding not only with dental substrate sites, but also with their hydrophilic diluent comonomers (Fig. 2.) and with free and bound water molecules remaining in acidetched tooth surfaces. Due to the hydrophilic ligand and hydroxyl groups, these molecules should be able to penetrate the hydrated layers of dentin and enamel and interact with collagen and tooth mineral. In this study, an attempt was made to have the solubility parameters of Type I collagen, the polymerizable cyclodextrin derivatives, and the diluent comonomers as nearly the same as possible so that the formulation components would not undergo phase separation during impregnation of the hydrated tooth components.

It is (or should be) common practice now to remove mechanically weak surface layers on teeth ("smear layers" and/or salivary pellicle) with an etching procedure. Smear layers can contain microorganisms and can impede the filling and sealing of dentinal tubules and their anastomosing or interconnected lateral canals with cross-linked adhesive polymers [5]. "Total etching" [6] usually comprises the brief application of an acidic solution, such as aqueous phosphoric acid, to remove debris from the tooth surfaces that require coverage with a dental resin. After this etching and a water rinse, the enamel has a superficially porous hydrated surface layer; the surface of dentin consists primarily of a thin layer of hydrated Type I collagen fibrils, somewhat denatured, that are retained by partial embedment in calcium phosphate minerals.

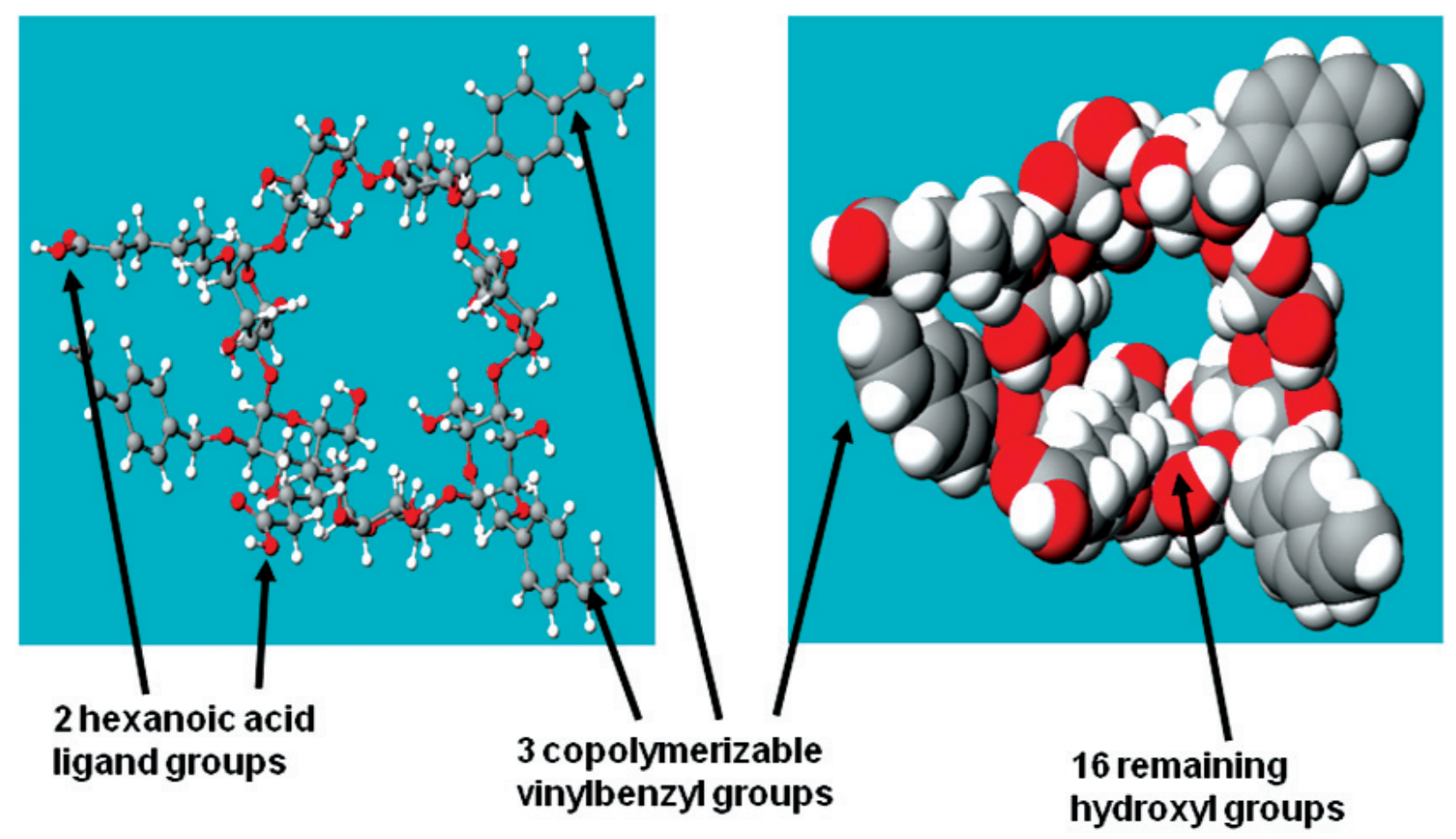

Fig. 1. Computer simulation of a polymerizable cyclodextrin derivative (PCD) with a ball-and-stick depiction on the left and a space-filling image on the right. Gray corresponds to carbon atoms, white to hydrogen atoms, and red to oxygen atoms. 


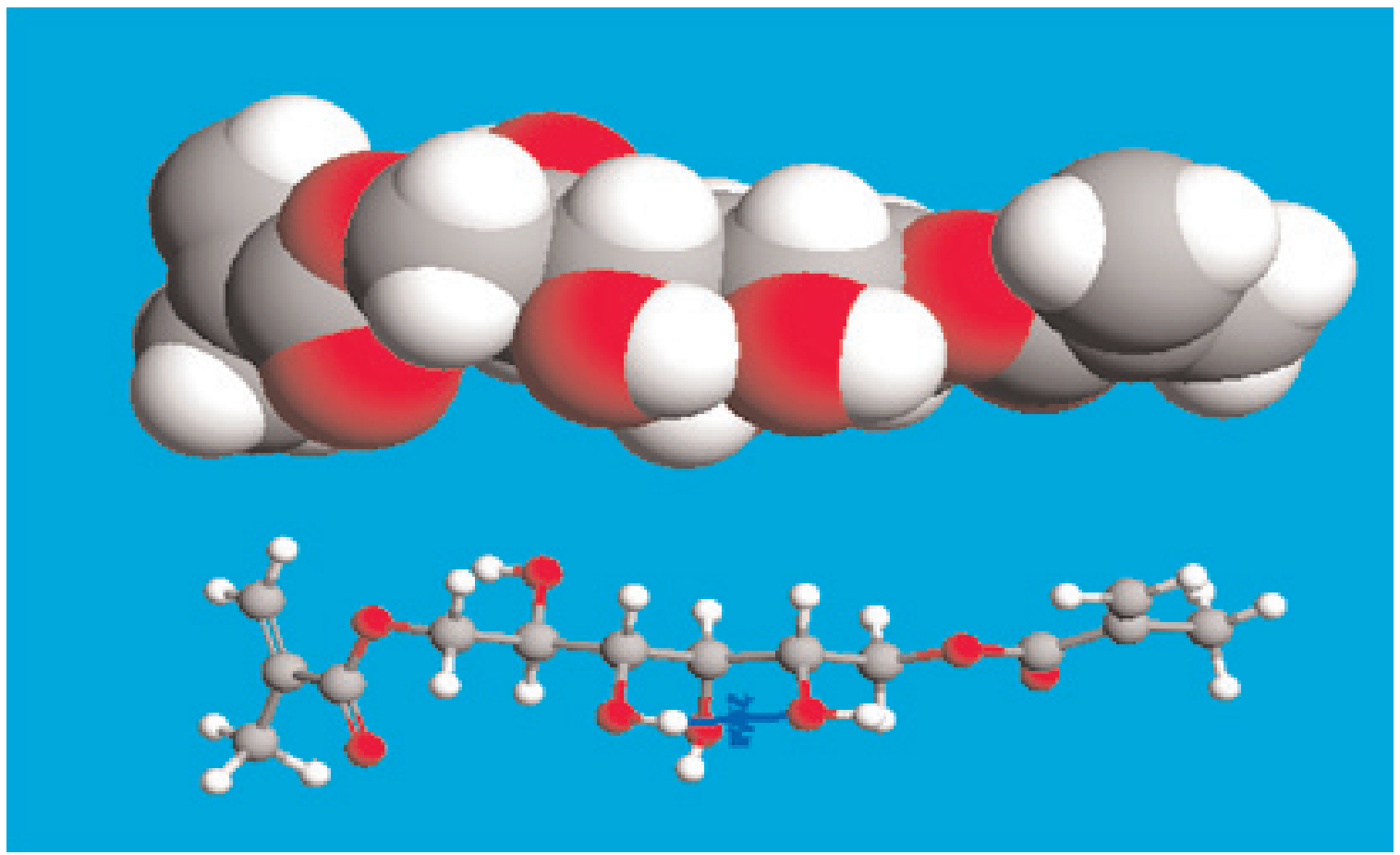

\section{Sorbitol dimethacrylate}

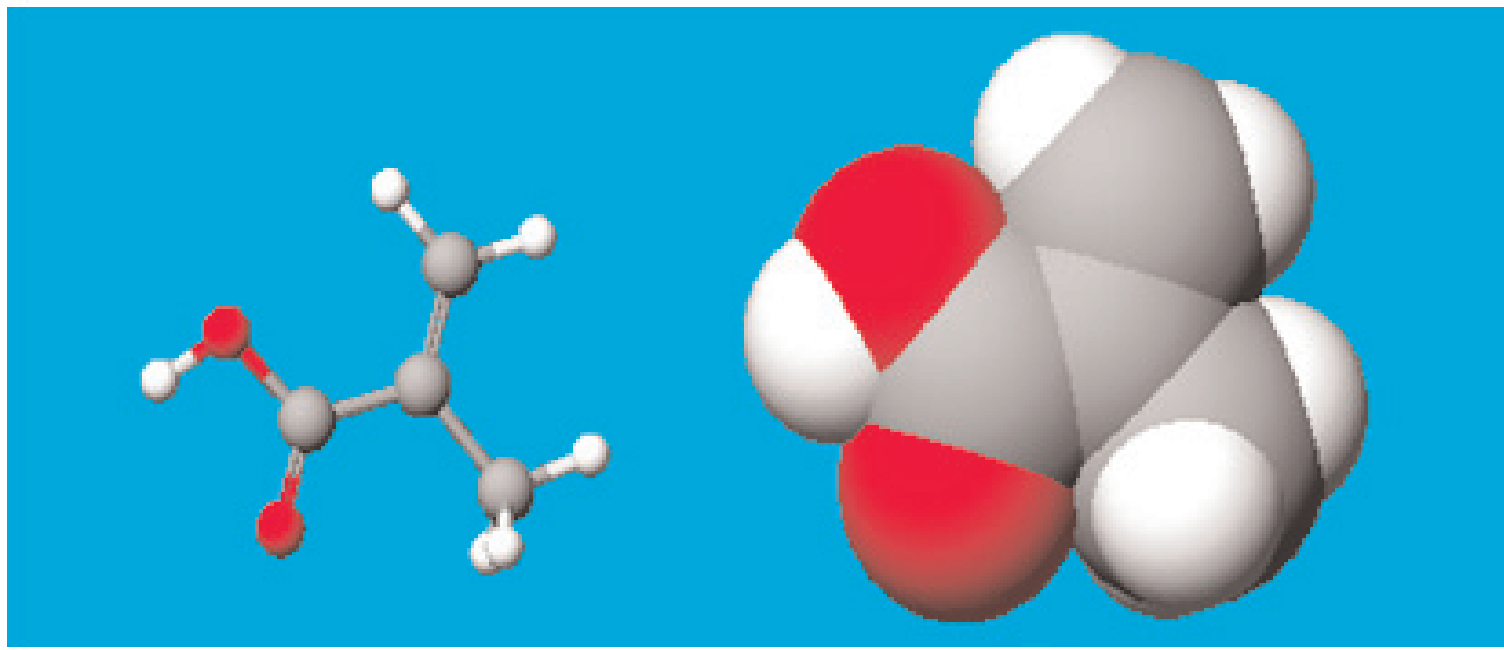

\section{Methacrylic acid}

Fig. 2. Sorbitol dimethacrylate and methacrylic acid reactive diluents used in the composition of bonding formulations one and two. Gray corresponds to carbon atoms, white to hydrogen atoms, and red to oxygen atoms. 
The wet porous enamel and the hydrated collagen network are the substrates to which adhesion must be achieved if good bonding is to be expected. Success strongly depends on the following factors: Complete and simultaneous interpenetration of all of the adhesion-promoting formulation components into the hydrated pores of enamel and throughout the waterfilled intact or denatured fibrillar collagen network of dentin and cementum, including interaction with underlying mineral. It seems reasonable to assume that the stoichiometric and physical forms of the original mineral(s) could have been changed by the acidic debridement and/or exposure to acidic monomers. Furthermore, there must be successful displacement of most of the water, by virtue of strong physicochemical affinity of the adhesion-promoting molecules, from the diverse "receptor sites" of these substrates. Formulations containing polymerizable cyclodextrin derivatives such as are reported here (especially improved versions thereof) are good candidates for fulfilling these requirements.

Cyclodextrins, especially appropriate derivatives thereof, comprise cyclic oligomers of glucose that can form water-soluble inclusion complexes with small molecules and portions of large compounds [7]. The synthesis of the polymerizable cyclodextrin derivatives used in this report is described elsewhere [4]. Briefly, these polymerizable cyclodextrin derivatives comprise beta-cyclodextrin molecules derivatized to attach combinations and permutations of both hydrophobic (organophilic) polymerizable groups and hydrophilic ligand groups on the members of a "family" of molecules. These molecules, with the groups attached by hydrolytically stable ether linkages, are based on cyclic oligosaccharides of seven glucopyranose units with alpha 1-4 glycoside linkages. The three-dimensional structures of beta-cyclodextrin molecules resemble truncated cones with hydrophobic interiors and hydrophilic exteriors that can form water-soluble inclusion complexes with small molecules and portions of large compounds [7]. Polymerizable cyclodextrin derivatives are water soluble because of the hydrophilic ligand groups and what remain of the original 21 hydroxyl groups that did not become derivatized. Although these quasi-spheroidal molecules have molecular weights mostly in the range of 1400 to 2000 grams per mole, three-dimensional computer modeling shows that they have diameters approximately the same as the cross-section of a single triplehelical collagen molecule (in the neighborhood of about $2 \mathrm{~nm}$ as estimated from the computer modeling).
This is much smaller than the sizes of the observable pores in the surfaces of acid-etched enamel, the internal diameters of dentinal tubules or their lateral canals, or the spaces between the collagen fibrils presenting in intertubular dentin after acid-etching and rinsing with water, according to observations made by staff members here.

The hypothesis in this study is that these types of polymerizable cyclodextrin derivative molecules, formulated with hydrophilic cross-linking diluent comonomers, can penetrate the hydration layers and form stronger adhesive bonds to hydrated dentin when compared with the control formulations.

\section{Materials and Methods}

\subsection{Shear Bond Adhesion Test Method}

Flat dentin surfaces were prepared by cutting off, with running water as a coolant, the tips of crowns of caries-free human molars with a low-speed diamond saw (Isomet; Buehler Ltd., Lake Bluff, IL, USA). The teeth were embedded with cold-curing resin in poly-carbonate holders, and the mid-coronal dentin surfaces were ground perpendicular to the long axis of the tooth on water-washed No. 320 grit $\mathrm{SiC}$ paper until the occlusal enamel was completely removed. For all except the commercial control bonding formulation, the dentin surfaces were etched (37\% phosphoric acid gel, Kerr Gel Etchant) for $15 \mathrm{~s}$ and rinsed with distilled water for $10 \mathrm{~s}$. Excess water was patted off with a damp cellulose tissue to prevent drying completely.

The sources for the bonding formulation components that were used are given in Table 1. Formulations were applied with a brush-tipped applicator for $20 \mathrm{~s}$, blown "dry" with an air stream for $10 \mathrm{~s}$ resulting in a glossy finish. The surfaces were light cured for $10 \mathrm{~s}$ with a halogen-type light (Dentsply Caulk Spectrum ${ }^{\mathrm{TM}}$ Curing Light). A stainless-steel ring having a having a $4 \mathrm{~mm}$ diameter central iris opening was held against the treated surface and a composite material (Prodigy Unidose P-C2, item No. 25886, Exp. 0498, “505096”) was condensed into the opening, with care taken to not overlap the top of the iris. The composite was light-cured for $60 \mathrm{~s}$ and then the assembly was left to stand for $5 \mathrm{~min}$, to allow for cooling after the exothermic polymerization of the monomers, before the holder was removed. Then the iris containing the bonded composite was soaked in distilled water for $24 \mathrm{~h}$ at about $23^{\circ} \mathrm{C}$ before bond testing in the shear mode. 
Volume 114, Number 1, January-February 2009

Journal of Research of the National Institute of Standards and Technology

Table 1. Materials used in the polymerizable cyclodextrin derivative (PCD) adhesive-bonding formulation

\begin{tabular}{|c|c|c|c|}
\hline Acronym & Chemical & Lot & Manufacturer. \\
\hline PCDs & $\begin{array}{l}\text { Family of polymerizable } \\
\text { cyclodextrin derivatives }\end{array}$ & B161-12 & Synthesized \\
\hline $\operatorname{Irganox}^{\circledR} 1330$ & $\begin{array}{l}\text { 1,3,5-trimethyl-2,4,6- } \\
\text { tris(3,5-di-(tert)-butyl-4- } \\
\text { hydroxybenzyl)benzene }\end{array}$ & 11107 & $\begin{array}{l}\text { Ciba Specialty Chemicals Corp., } \\
\text { Tarrytown, NY } 10591\end{array}$ \\
\hline SDM2 & sorbitol dimethacrylate & $\begin{array}{l}9676 \\
18-6-1\end{array}$ & $\begin{array}{l}\text { Monomer-Polymer } \\
\& \text { Dajac Laboratories, Inc. } \\
\text { Feasterville, PA } 19053\end{array}$ \\
\hline MAA & methacrylic acid & 09610HB & $\begin{array}{l}\text { Sigma-Aldrich, Inc., } \\
\text { St. Louis, MO } 63103\end{array}$ \\
\hline Irgacure $^{\circledR} 819$ & $\begin{array}{l}\text { Phenyl bis- } \\
\text { (2,4,6-trimethylbenzoyl)- } \\
\text { phosphine oxide }\end{array}$ & 21J887S & $\begin{array}{l}\text { Ciba Specialty Chemicals Corp., } \\
\text { Tarrytown, NY } 10591\end{array}$ \\
\hline
\end{tabular}

A holding device was used to evaluate the shear bond strengths. The stainless-steel ring containing the dentin-bonded composite within its iris was placed against a vertical surface of a nylon block. The ring and the composite were sheared off, at a crosshead speed of $0.5 \mathrm{~mm} / \mathrm{min}$, with a flat chisel pressing against the edge of the steel ring close to the tooth surface. The flat chisel was connected to the platen of a Universal Testing Machine (Instron Corp., Canton, MA).

\subsection{Experimental Bonding Formulation 1}

In the experimental formulation 1 , a portion $(0.0419 \mathrm{~g})$ of the family of polymerizable cyclodextrin derivative reaction products $[3,4]$ was mixed with sorbitol dimethacrylate (0.3261 g "SDM2") and methacrylic acid (0.2383 g MAA) as reactive diluents to lower the polymerizable cyclodextrin derivative viscosity. Phenylbis[2,4,6-trimethylbenzoyl]phosphine oxide (0.0271 g Irgacure $\left.{ }^{\circledR} 819\right)$ was added to serve as the photoinitiator (Fig. 3.) to induce polymerization. The formulation also contained a very small amount of the antioxidant $\operatorname{Irganox}^{\circledR} 1330$ plus the stabilizers contained in the sorbitol dimethacrylate and methacrylic acid as received. The composite was applied and the shear bond adhesion test method for Formulation 1 was performed as described in Sec. 2.1, with $n=6$, where $n$ is the number of independent samples used for the shear bond strength measurements.

\subsection{Comparative Bonding Formulation 2}

The same formulation 1 without the family of polymerizable cyclodextrin derivative reaction products, maintaining the photoinitiator concentration approximately constant, was also prepared to isolate the effect of the presence of the polymerizable cyclodextrin derivatives. This formulation contained $0.3261 \mathrm{~g}$ of SDM2, $0.2383 \mathrm{~g}$ of methacrylic acid, $0.0271 \mathrm{~g}$ of Irgacure $^{\circledR} 819$, and the same small amount of Irganox ${ }^{\circledR}$ 1330 plus the stabilizers contained in the SDM2 and methacrylic acid as received. The composite was applied and the shear bond adhesion test method was the same as that described in Sec. 2.1, with $n=9$.

\subsection{Control Bonding Formulation 3}

A commercially-available self-etching primer bonding system was used as a control (Clearfil Protect Bond Primer No.1, lot 0004A, and Clearfil Protect Bond, Kuraray Med. Inc., Kurashiki, Okayama, Japan, Lot 00008A). Components listed in a Material Safety Data Sheet for "Bond Liquid, Clearfil Protect Bond" are as follows: "Silanated colloidal silica, sodium fluoride, bisphenol A diglycidylmethacrylate, 2-hydroxyethyl methacrylate, hydrophobic dimethacrylate, 10-methacryloyloxydecyl dihydrogen phosphate, N,N-diethanolp-toluidine, and d,l-camphorquinone" [8]. The manufacturer's instructions were followed: The abraded dentin surface was rinsed with distilled water for $10 \mathrm{sec}$ and then excess water was patted off with a damp 


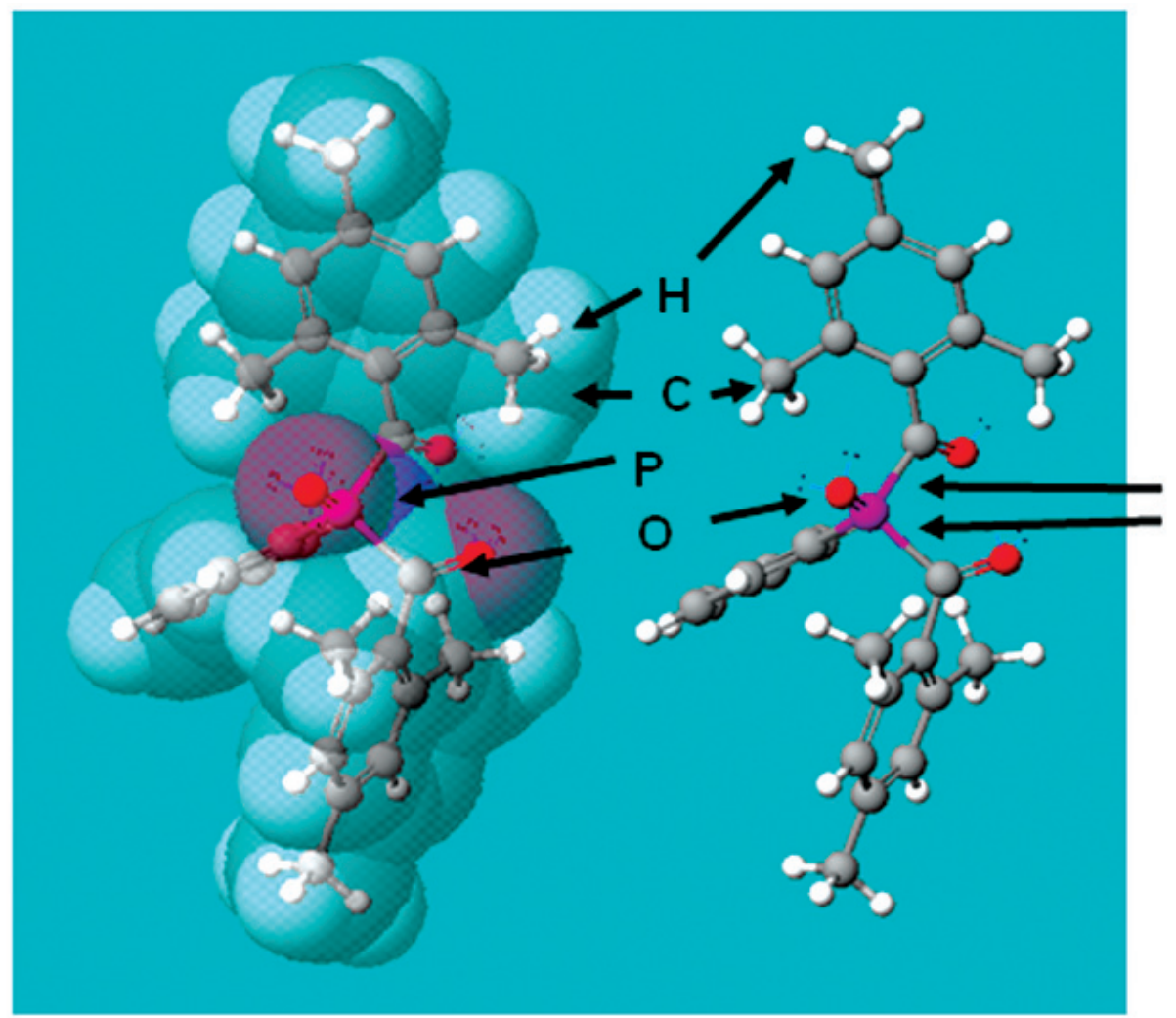

\section{It is the bonds attached to the phosphorous atom that photo dissociate to form free radicals.}

Fig. 3. Photoinitiator used in the experimental formulations: phenyl bis(2,4,6-trimethylbenzoyl) phosphine oxide. These molecules are too large to become totally complexed ("cloistered," encapsulated) within the hydrophobic core spaces of polymerizable cyclodextrin derivatives. However, in an increasingly aqueous environment during penetration of surface hydration layers, the aromatic side groups would be expected to become sufficiently complexed to become surrounded by hydrophilic PCD molecules to enable diffusion and accompaniment of the comonomers to reach the unaltered substrate minerals. Gray corresponds to carbon atoms, white to hydrogen atoms, red to oxygen atoms and purple phosphorous atoms.

cellulose tissue without completely drying. The self-etching primer was gently applied, mildly dried with the airflow, and then covered with the Protect Bond. The solvent was removed with the airflow, and then the coated surface was light-cured for $10 \mathrm{~s}$. The composite was placed as described in Sec. 2.1 and lightcured for $60 \mathrm{~s}$. The shear bond adhesion test method was also the same as that described in Sec. 2.1, with $n=6$.

\subsection{An Alternative Bonding Procedure With Formulation 1}

An exploratory comparison was made with the experimental formulation 1 (B161-136 family of polymerizable cyclodextrin derivatives plus sorbitol dimethacrylate plus methacrylic acid plus Irgacure ${ }^{\circledR} 819$, with the difference that after the etching, rinsing, and removal of excess water with a damp cellulose tissue, 200-proof ethanol was applied to the dentin surface with a brush-tipped applicator for $10 \mathrm{~s}$ to replace much of the surface water with alcohol before application of the experimental formulation [9]. Otherwise, the procedure was as in Sec. 2.1, with $n=3$.

\subsection{An Alternative Bonding Procedure With Formulation 2}

The formulation and testing procedure were the same as in Sec. 2.3, without the family of polymerizable cyclodextrin derivative reaction products and maintaining the photoinitiator concentration approximately constant, except that after the excess water was removed from the surfaces, 200-proof ethanol was 
applied to the dentin surfaces with a brush-tipped applicator for $10 \mathrm{~s}$ to replace much of the surface water with alcohol [9]. The shear bond adhesion test method was the same as that described in Sec. 2.1, with $n=3$.

\subsection{Another Alternative Bonding Procedure With Formulation 1}

This comparison was made as described in Sec. 2.1 with the differences being that after the excess water was patted off, 200-proof ethanol was applied to the dentin surfaces with a brush-tipped applicator for $10 \mathrm{~s}$ to replace much of the surface water with alcohol [9], the experimental formulation (B161-136 family of polymerizable cyclodextrin derivatives plus sorbitol dimethacrylate plus methacrylic acid plus Irgacure ${ }^{\circledR}$ 819) as described in Sec. 2.2 was applied with a brushtipped applicator for $20 \mathrm{~s}$, blown "dry" with an air stream for $10 \mathrm{~s}$ resulting in a glossy finish. Additionally, this was then covered with Clearfil Protect Bond. The solvent was removed with the airflow, and then the coated surface was light cured for $10 \mathrm{~s}$. Otherwise, the procedure was as in Sec. 2.1, with $n=3$.

\subsection{Statistics}

One-way analysis of variance (ANOVA) was performed on the results to detect significant effects. Newman-Keuls multiple comparison tests were conducted with the data of Secs. 2.2, 2.3, and 2.4 to determine if a significant difference $(p<0.05)$ existed where $p$ is a fraction of the probability distribution.

\subsection{Scanning Electron Microscopy}

After the shear bond adhesion tests, some of the teeth were cut longitudinally and treated to evaluate the hybrid layer. Two methods were used: $30 \%$ phosphoric acid etching for $15 \mathrm{~s}$ and bleaching with $6.15 \%$ sodium hypochlorite (Ultra Clorox ${ }^{\circledR}$ ) for $8 \mathrm{~min}$, or polishing and argon-ion etching for $5 \mathrm{~min}$.

\section{Results}

\subsection{Adhesion Tests}

The shear bond strength average values, defined as the load at fracture divided by the iris opening areas, are given in Table 2. The average shear bond strength of formulation 1 (Sec. 2.2) containing the family of PCD reaction products was significantly higher $(p<0.05)$ than that of formulation 2 (Sec. 2.3), which was the same formulation minus the family of PCD reaction products, indicating that the presence of polymerizable cyclodextrin derivatives led to improved bonding. The average shear bond strength of formulation 1 (Sec. 2.2) ranked higher than that of formulation 3 , the commercial control (Sec. 2.4). The preliminary application of alcohol in procedure Sec. 2.5 did not increase the average bond strength with formulation 1 in comparison with that described in Sec. 2.2. The preliminary application of alcohol in procedure Sec. 2.6 with formulation 2 did not increase the average bond strength relative to formulation 1 in procedure Sec. 2.2, but its mean bond strength ranked higher than its value with procedure Sec. 2.3. Procedure Sec. 2.7 did not give an increase in average bond strength relative to that obtained with procedure Sec. 2.2.

\subsection{Statistics}

One-way ANOVA analysis found that the type of formulation used had a significant effect $(p<0.05)$ on the shear bond strength values obtained with procedures Sec. 2.2 and Sec. 2.3.

\subsection{Scanning Electron Microscopy}

The formulation 1 containing the derivatized beta cyclodextrins (2.2) and the formulation 2 not containing the family of polymerizable cyclodextrin derivatives (2.3) resulted in mostly cohesive failures within the resinous materials, with the areas of separation approximately the same as the areas of the iris opening, as observed with a binocular measuring microscope

Table 2. Shear bond strength (SBS) average values in $\mathrm{MPa}$ (where $1 \mathrm{~Pa}=1 \mathrm{~kg} \cdot \mathrm{m}^{-1} \cdot \mathrm{s}^{-2}$ ), defined as the load at fracture divided by the iris opening areas. The estimates of standard uncertainty are indicated by the standard deviation (SD) values.

\begin{tabular}{lcccccc}
\hline \hline $\begin{array}{l}\text { Formulation } \\
\text { Procedure }\end{array}$ & $\begin{array}{c}1 \\
2.2(n=6)\end{array}$ & $2.3(n=9)$ & $2.4(n=6)$ & $2.5(n=3)$ & $2.6(n=3)$ & $2.7(n=3)$ \\
\hline SBS in MPa (SD) & $24.6(5.4)$ & $12.4(3.7)$ & $17.4(4.0)$ & $22.7(4.6)$ & $19.2(3.8)$ & $22.7(6.7)$ \\
\hline
\end{tabular}


(Model MZ16 Stereo-optical microscope system, with traveling stage, by Leica Microsystems, Wetzlar, Germany). The commercial control formulation 3 (described in Sec. 2.4) failed mostly at its adhesive interface with dentin (Fig. 4), and the areas of adhesive separation tended to be larger than the areas of the iris opening.

\section{Discussion}

Reported shear bond strength values vary widely, depending in part on the selected test procedures [10]. Therefore, numerical values should be compared only within the method that is used.

Although only about a maximum of seven hydroxyl groups in molecules of this family of polymerizable cyclodextrin derivatives were substituted, the bonding formulation prepared with this family of reaction products did result in an average shear bond strength significantly higher than that of the analogous formulation that did not contain this family of polymerizable cyclodextrin derivatives. One of the important distinctions of this polymerizable cyclodextrin derivative family was that the synthetic procedure attached both the polymerizable groups and the terminal carboxylate ligand groups to the beta-cyclodextrin molecules with hydrolytically stable covalent ether linkages. There is reason to believe that some of the instability associated with contemporary adhesive resins is related to hydrolysis and/or saponification of ester linkages within the interfacial bonding polymers $[11,12]$. For reasons of expediency, the diluent cross-linking comonomer sorbitol dimethacrylate was used in the present study even though it contained potentially hydrolyzable ester linkages. The preparation and evaluation of "sorbitoldivinylbenzyl ether" is planned for use as an alternative.

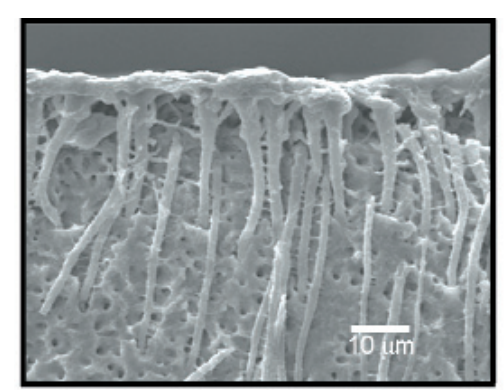

Formulation 1

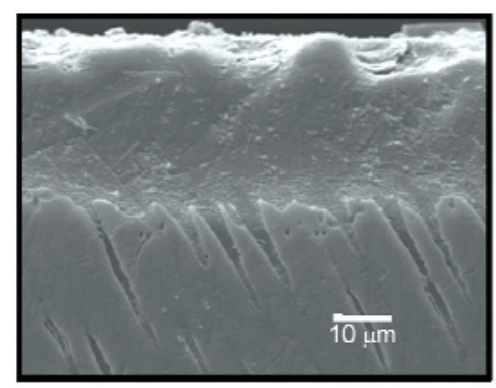

Formulation 1

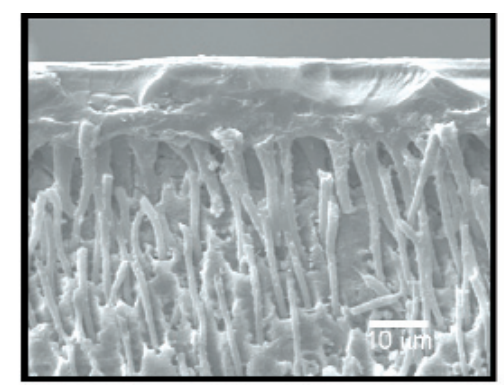

Formulation 2

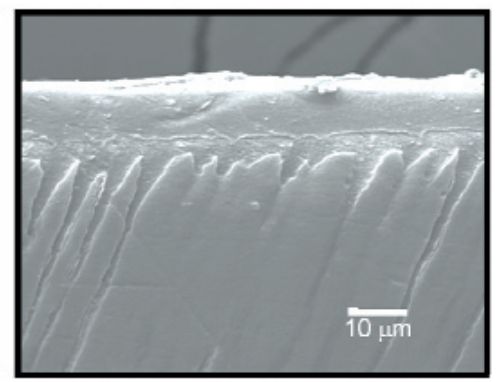

Formulation 2

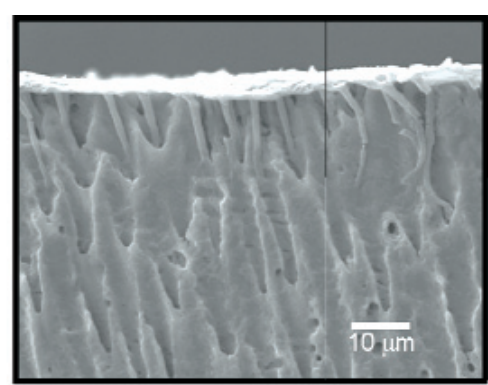

Formulation 3

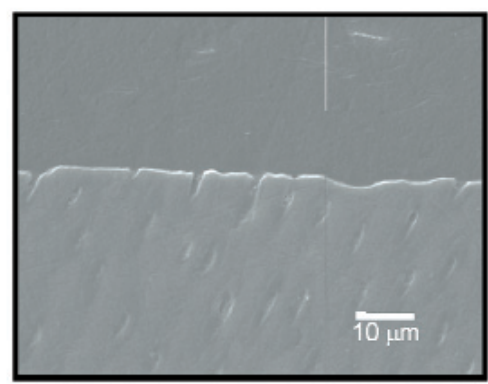

Formulation 3

Fig. 4. SEM pictures of debonded teeth: These pictures showed evidence of hybrid layer formations in the case of Formulations 1 and 2 . For the control (Formulation 3) a hybrid layer could not be detected. The upper image of the Formulation 1 sample shows continuity of the hybrid layer with the polymer in the tubules and in the lateral canals. The upper images are of samples that had been acid etched for $15 \mathrm{~s}$ and bleached for $8 \mathrm{~min}$. The lower images are of samples that had been argon-etched. The white bars represent 10 micrometers. 
Another important distinction was that the vinylbenzyl groups should have facile free-radical polymerization charactistics similar to that of styrene. The vinyl double bonds of styrene readily homopolymerizes and copolymerizes with the double bonds of methacrylate esters. However, the stable covalent attachment of the vinylbenzyl groups to such large hydrophilic molecules as polymerizable cyclodextrin derivatives should obviate the biological incompatibility of styrene, as such, in dental formulations. However, it is essential, of course, that all new formulations, including the novel compositions described herein be tested and evaluated in a manner that assures their safety for use in dental or bio-medical applications.

This study does not address the intimately related problem of the polymerization shrinkage stresses that develop primarily during the cooling that follows the exothermic polymerization of composite materials and other dental resins [13-17]. However, this study together with the investigation results of many others have introduced novel concepts for modifications of compositions relevant to lessening the stresses that composites and adhesion-promoting formulations currently generate at tooth-polymer interfaces. Investigation of these concepts is planned.

\section{Conclusions}

These preliminary results [18] are in accordance with the hypothesis that polymerizable cyclodextrin derivative molecules, formulated with hydrophilic cross-linking diluent comonomers, can penetrate the hydration layers and form strong adhesive bonds to hydrated dentin as compared to the controls. The hypothesis was supported. However, improved synthetic methods are underway to obtain more than one polymerizable group on substantially every reaction product molecule to provide densely cross-linked polymers, and to obtain an increased number of ligand groups on these molecules to enable an increased number of binding interactions with substrate "receptor sites." It is important that the water-soluble polymerizable cyclodextrin derivative molecules can transport water-insoluble polymerization initiators by means of complexation, together with the hydrophilic cross-linking diluent comonomers, through surface hydration layers to form strong adhesive bonds.

\section{Acknowledgements}

This work was supported through funding from the National Institute for Craniofacial and Dental Research, Grant 2 R01 DE005129-26A2, and in part by the American Dental Association Foundation and the National Institute of Standards and Technology. We also thank Elena F. Burguera for her significant contributions toward the development of this report, Jennifer Moreau for help in analyzing MALDI-TOF MS data; Dr. David VanderHart (NIST) for help in analyzing the NMR spectra, and Dr. Sabine Dickens and Dr. William E. Wallace for helpful discussions.

\section{Disclaimer}

Certain commercial materials and equipment are identified to specify experimental procedures. In no instance does such identification imply recommendation by NIST or the ADA Foundation or that the material identified is necessarily the best available for the purpose.

Furthermore, it is essential that all materials described hereinabove be tested using methods described in ISO standards and ADA/ANSI specifications and/or otherwise evaluated in a manner that assures their safety for use in dental, biomedical, or industrial applications as appropriate.

\section{References}

[1] I. A. Mjör, Clinical diagnosis of recurrent caries, J. Amer. Dent. Assoc. 136 (10), 1426-1433 (2005)

[2] I. A. Mjör, Placement and replacement of restorations, Oper. Dent. 6, 49-54 (1981).

[3] R. L. Bowen, E. F. Burguera, K. M. Flynn, C. M. Guttman, and W. E. Wallace, Synthesis of polymerizable cyclodextrin derivatives (PCDs) and characterization with MALDI-TOF-MS, J. Dent. Res. Vol. 86, Special Issue A, Abstr. 0114, IADR (2007).

[4] R. L. Bowen, G. E. Schumacher, C. M. Carey, A. A. Giuseppetti, K. M. Flynn, and C. M. Guttman, Synthesis of polymerizable cyclodextrin derivatives for use in adhesionpromoting monomer formulations, J. Res. Natl. Inst. Stand. Technol. 114 (1), 1-9 (2009).

[5] D. H. Pashley and R. M. Carvalho, Dentine permeability and dentine adhesion, Journal of Dentistry, 25 (5), 355-372 (1997).

[6] T. Fusayama, Total etch technique and cavity isolation, J. Esthet. Dent. 4 (4),105-109 (1992).

[7] M. E. Davis and M. E. Brewster, Cyclodextrin-based pharmaceutics: past, present and future, Nat. Rev. Drug Discov. Dec. 3 (12), 1023-1035 (2004). 
[8] http://www.tinmandental.com/msds/Clearfil_Protect_Bond_ bond_(04_2006).pdf; 1913MSDS 04/06.

[9] F. R. Tay, D. H. Pashley, R. R. Kapur, M. R. O. Carrilho, Y. B. Hur, L. V. Garrett, and K. C. Y. Tay, Bonding BisGMA to dentin-a proof of concept for hydrophobic dentin bonding, J. Dent. Res. 86 (11), 1034-1039 (2007).

[10] S. H. Dickens and M. F. Milos, Relationship of dentin shear bond strengths to different laboratory test designs, American Journal of Dentistry 15 (3), 185-192 (2002).

[11] B. A. Lin, F. Jaffer, M. D. Duff, Y. W. Tang, and J. P. Santerre, Identifying enzyme activities within human saliva which are relevant to dental resin composite biodegradation, Biomaterials 26, 4259-4264 (2005).

[12] S. R. Armstrong, J. L. P. Jessop, M. A. Vargas, Y. Zou, F. Qian, J. A. Campbell, and D. H. Pashley, Effects of exogenous collagenase and cholesterol esterase on the durability of the resin-dentin bond, J. Adhes. Dent. 8 (3), 151-160 (2006).

[13] R. L. Bowen, Adhesive bonding of various materials to hard tooth tissues, VI. forces developing in direct-filling materials during hardening. J. Amer. Dent. Assoc.74 (3), 439-445 (1967).

[14] R. L. Bowen, Adhesive bonding of various materials to hard tooth tissues: forces developing in composite materials during hardening, J. Amer. Dent. Assoc. 106, 475-477 (1983).

[15] J. W. Park and J. L. Ferracane, Residual stress in composites with the thin-ring-slitting approach, J. Dent. Res. 85, 945-949 (2006).

[16] H. Lu, J. W. Stansbury, and C. N. Bowman, Towards the elucidation of shrinkage stress development and relaxation in dental composites, Dental Materials 20 (10), 979-986 (2004).

[17] A. J. Feilzer and B. S. Dauvillier, Effect of TEGDMA/BisGMA ratio on stress development and viscoelastic properties of experimental two-paste composites, J. Dent. Res. 82 (10), 824 828 (2003).

[18] E. F. Burguera, R. L. Bowen, G. E. Schumacher, and A. A. Giuseppetti, Shear bond strength of a polymerizable cyclodextrin derivative (PCD) formulation, J. Dent. Res. Vol. 86, Special Issue A, Abstr. No. 0344, (2007).

About the authors: Ray Bowen is a dentist who invented composite dental restorative materials while working as an ADA Research Associate at NBS in 1956; he has since continued improving adhesive bonding materials for composites in the PRC group within the Dental and Medical Materials Program. Dr. Schumacher is the PRC Associate Director and Chief Research Scientist of the PRC Clinical Dentistry Program. Mr. Giuseppetti is a Laboratory Technician in the Clinical Program. Dr. Carey conducts independent research and is Grant Administrator in the PRC's Office of the Director. Dr. Guttman is in the Characterization and Measurement Group within the NIST Polymers Division of MSEL. The National Institute of Standards and Technology is an agency of the U.S. Department of Commerce. 